\title{
Complexes of 3.6 kDa Maltodextrin with Some Metals
}

\author{
Piotr Tomasik ${ }^{1, *}$, James W. Anderegg ${ }^{2}$ and Christopher H. Schilling ${ }^{3}$ \\ ${ }^{1}$ Department of Chemistry, University of Agriculture, Mickiewicz Ave., 21, 31120 Cracow, Poland. \\ Tel/Fax (+48) 12-662-43-35. \\ 2 Ames Laboratory, Ames, IA 50011, U.S.A. \\ 3 Department of Mechanical Engineering, Saginaw Valley State University, University Center, MI \\ 48710, U.S.A.
}

*Author to whom all correspondence should be addressed; e-mail: rrtomasi@cyf-kr.edu.pl

Received: 30 October 2003; in revised form: 17 May 2004 / Accepted: 25 May 2004 / Published: 30 June 2004

\begin{abstract}
Preparation of magnesium, lanthanum, and bismuth(III) complexes of $3.6 \mathrm{kDa}$ maltodextrin and some properties of the resulting materials are presented. The metal derivatives contain metals bound to the oxygen atoms of the hydroxyl groups of maltodextrin. Additionally, the metal atoms are coordinated to the hydroxyl groups of the D-glucose units of the macroligand. Such coordination stabilized the metal - oxygen bond against hydrolysis, even in boiling water. The presence of magnesium and lanthanum atoms increased the thermal stability of maltodextrin, whereas bismuth atoms decreased it.
\end{abstract}

Keywords: Biopolymer, bismuth(III), lanthanum, magnesium, polysaccharides, x-ray photoelectron spectroscopy.

\section{Introduction}

For over a decade, research has been developed on the utilization of polysaccharides as versatile, biodegradable, renewable sources for chemical industry, replacing mineral resources such as petroleum, coal, and metal ores [1]. There are three main paths leading to the targets. First, 
polysaccharides can be a source of energy via their direct combustion or their composting into biomass as a source of biogas. Second, polysaccharides are a source of carbon monoxide, methanol, ethylene, methanal, and so on, which are fundamental petrochemicals. Finally, polysaccharides and products resulting from their chemical modifications can be utilized directly in several branches of industry.

Recently, several papers on the application of maltodextrins as environmentally benign plasticisers in aqueous colloidal processing of ceramics have been published [2-6]. In this function they replace other environmentally hazardous plasticizers of mainly petrochemical origin. Like other plasticisers, maltodextrins prevent agglomeration of ceramic metal oxide based powders, provide a compaction of the ceramic powder to high packing-density ceramic pastes, alleviate cracks and warpage during subsequent kiln firing, and also offer a high degree of moldability (plasticity) in shape-forming operations [2-6].

Moreover, in order to achieve desirable end-properties of ceramic materials, other metal oxides are added in microscale amounts to the major constituent. Examples of these are, for instance, $\mathrm{MgO}$ [7], $\mathrm{La}_{2} \mathrm{O}_{3}$ [8] and $\mathrm{Bi}_{2} \mathrm{O}_{3}$ [9]. There are some problems associated with introducing these dopants in granular form. The dopant grains can be too large, in which case the solid-state reaction does not go to completion. Additionally, it is difficult to achieve a uniform spatial distribution of the dopant in the ceramic product. In this case, extremely long sintering times would be needed to homogenise the product. Making use of the ability of polysaccharides to form metal complexes could provide for simultaneous plasticising and doping of ceramic cakes. Polysaccharides form various types of compounds and complexes with metal compounds. Depending on the metal, either sorption complexes or Werner-type complexes can be formed. In both cases no covalent bonds are involved in bonding $[10,11]$. Apart from sorption and Werner complexes, complexes are known where covalently bound metal atoms are additionally coordinated to numerous sites of ligation, e.g. hydroxyl groups of the D-glucose units of starch. Such compounds are available through various reactions, among others, via the reaction of starch with metal alkoxides [12-16]. Also, the reactions of starch with certain metal chlorides [17] and metals [18] provide the corresponding starch-metal complexes.

In this paper the synthesis, structure and properties of magnesium, lanthanum, and bismuth(III) derivatives of $3.6 \mathrm{kDa}$ maltodextrin are described. Because such compounds could potentially serve as dopants and plasticisers for micrometric metal oxide based ceramics $3.6 \mathrm{kDa}$ maltodextrin was selected for derivatisation. The usefulness of $3.6 \mathrm{kDa}$ maltodextrin as a binder and plasticiser for micrometric metal oxide-based ceramics has been proven in several instances [6].

\section{Results and Discussion}

Table 1 presents the results of the thermogravimetric analyses. They serve to rationalize the following interpretation of the reaction with the bismuth reagents. When bismuth tri(tert-pentoxide) and the D-glucose unit $\left.\left[(\mathrm{HO})_{2}-\mathrm{Glc}-\mathrm{OH}\right)\right]$ were taken in the $1: 1$ proportion they could react intramolecularly with involvement of either one or two of its alkoxide functions. In this manner either compounds $\mathbf{1}$ or $\mathbf{2}$ would be formed, respectively. 
(HO) ${ }_{2} \mathrm{GlC}-\mathrm{O}-\mathrm{Bi}\left(\mathrm{OC}_{5} \mathrm{H}_{11} \text {-tert }\right)_{2}$

(1)
HOGIC-O-Bi-OC ${ }_{5} \mathrm{H}_{11}$-tert

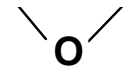

(2)
$(\mathrm{HO})_{2} \mathrm{GIC}-\mathrm{O}-\mathrm{Bi}-\mathrm{O}-\mathrm{GIC}(\mathrm{OH})_{2}$ O-C ${ }_{5} \mathrm{H}_{11}$-tert

(3)

In the same proportions the bismuth reagent might also react with the D-glucose units in an intermolecular manner, e.g. with involvement of two of its alkoxide functions to give 3. Upon heating, 2-methylbut-2-ene could be lost in the first step of decomposition of these compounds. This decomposition would be associated with the calculated weight losses reported in Table 1.

Table 1. Calculated and Found Weight Losses Associated with the First Step of Decomposition of Metal Derivatives of Maltodextrin

\begin{tabular}{|c|c|c|c|}
\hline \multirow{3}{*}{ Compound $^{\mathrm{a}}$} & \multicolumn{3}{|c|}{ Weight loss and residues (\%) } \\
\hline & \multicolumn{2}{|c|}{ Calculated for } & \multirow[b]{2}{*}{ Found $^{\mathrm{c}}$} \\
\hline & Alkene elimination ${ }^{\mathrm{b}}$ & Metal oxide residue & \\
\hline \multicolumn{4}{|c|}{$\operatorname{Bi}($ III) $:$ Maltodextrin $=1: 1$} \\
\hline 1 & 12.9 & & \\
\hline 2 & 15.35 & & \\
\hline 3 & 11.3 & & 11.5 \\
\hline \multicolumn{4}{|c|}{$\operatorname{Bi}(\mathrm{III}):$ Maltodextrin $=2: 1$} \\
\hline 4 & 10.5 & & \\
\hline 5 & 8.35 & & \\
\hline 6 & $8.06^{\mathrm{d}}$ & & 8.0 \\
\hline \multicolumn{4}{|c|}{ La $:$ Maltodextrin $=1: 1$} \\
\hline 7 & 8.6 & & \\
\hline 8 & 11.7 & & 11.8 \\
\hline 9 & 8.1 & & \\
\hline \multicolumn{4}{|c|}{ La $:$ Maltodextrin $=2: 1$} \\
\hline 10 & $12.5^{\mathrm{e}}$ & & \\
\hline 11 & $13.6^{\mathrm{e}}$ & & \\
\hline 12 & $8.15^{\mathrm{e}}$ & & 9.0 \\
\hline \multicolumn{4}{|c|}{$\mathrm{Mg}:$ Maltodextrin $=1: 1$} \\
\hline 13 & & 21.9 & 21.1 \\
\hline 14 & & 18.6 & \\
\hline 15 & & 11.6 & \\
\hline
\end{tabular}


Table 1. Cont.

\begin{tabular}{|l|l|l|l|}
\hline \multicolumn{3}{|c|}{ Mg: Maltodextrin = 2:1 } \\
\hline $\mathbf{1 6}$ & & 29.8 & \\
$\mathbf{1 7}$ & & 15.2 & \\
$\mathbf{1 8}$ & 20.1 & 20.8 \\
\hline
\end{tabular}

a The notation of compounds according to the text.

b The calculations for the elimination of one molecule of alkene.

c Found from the thermogram.

d Calculated for the loss of two molecules of 2-methylbut-2-ene. This is actually the 3:2 compound.

e Calculated for the loss of three molecules of propene.

The determined weight loss fits very well the value calculated for compound $\mathbf{3}$ resulting from intermolecular crosslinking of the maltodextrin chains with one molecule of the bismuth reagent. The compounds 4,5 or 6 could result from the reaction in which the bismuth reagent reacted with maltodextrin in a 2:1 proportion. Structures $\mathbf{4}$ and $\mathbf{5}$ result from the assumption that only one glucose unit reacted with two molecules of the bismuth reagent. Compound $\mathbf{6}$ would result from intermolecular crosslinking of two glucose units which, additionally, reacted with bismuth tri(tert-pentoxide). Also decomposition of each compound could proceed via evolution of 2-methylbut-2-ene. The relevant weight loss fits very well the value calculated for compound $\mathbf{6}$ (see Table 1).
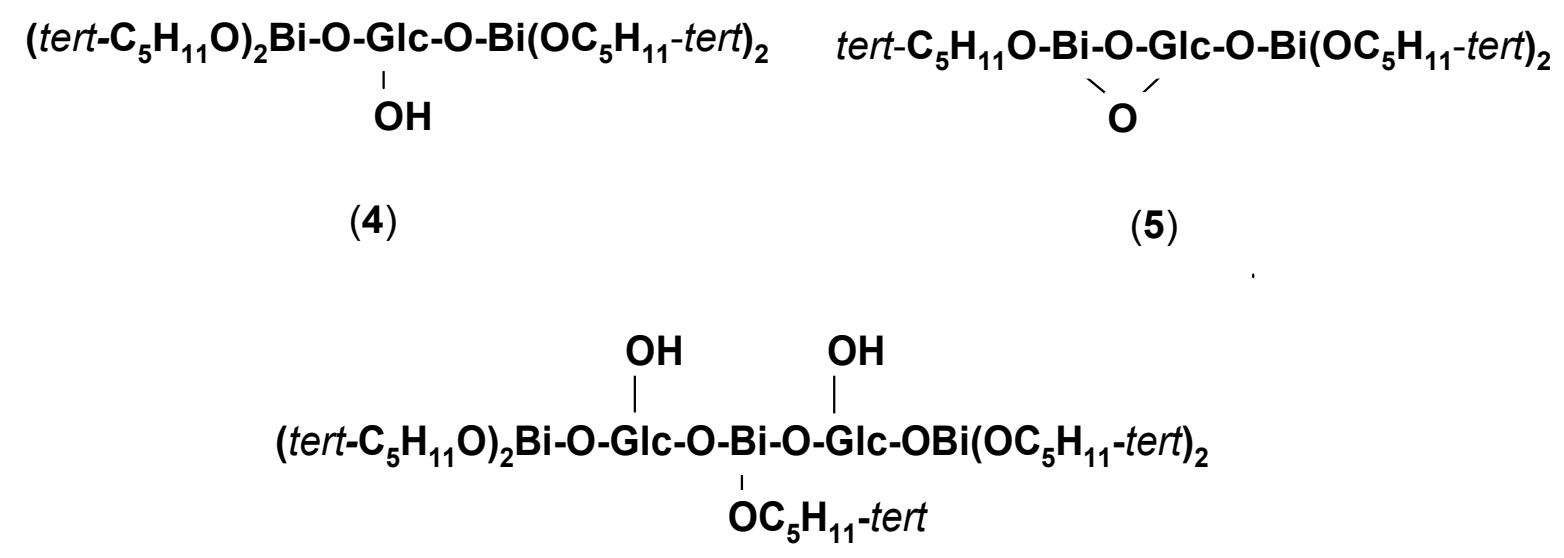

(6)

Lanthanum tri-isopropoxide should react with maltodextrin similarly to bismuth trialkoxide. Structures of resulting compounds resemble these of compounds 1-6. Reaction products in the 1:1 and 2:1 reaction mixtures are denoted in Table 1 as compounds 7-9 and 10-12, respectively. Every compound of these series could decompose on heating with evolution of propene (potential $\beta$-transelimination). In the group of compounds potentially available from the reaction in the 1:1 mixture the results of the thermogravimetric analysis corresponded fairly well to the value calculated for 
compound 8. The product $\mathbf{1 2}$ seemed to be the likely structure resulting from the reaction in the 2:1 blend as could be deduced from the weight loss in the relevant thermogram (Table 1). The weight loss associated with its thermal decomposition evolving three molecules of propene fits the calculated value satisfactorily. This compound resulted from intermolecular crosslinking of maltodextrin.

Because the methoxide moiety could not liberate any alkene on the thermolysis of magnesium maltodextrinates the elucidation of their structure was based on the amount of residual magnesium oxide after burning up the sample in the thermogravimetric crucible up to $1000{ }^{\circ} \mathrm{C}$. Results found for the reaction product with 1:1 composition corresponded satisfactorily to that calculated for structure $\mathbf{1 3}$ and did not fit results calculated for structures $\mathbf{1 4}$ and $\mathbf{1 5}$.

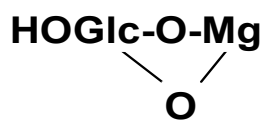

(13)

\section{$(\mathrm{HO})_{2} \mathrm{GlC}-\mathrm{O}-\mathrm{Mg}-\mathrm{OCH}_{3}$}

(14)
$(\mathrm{HO})_{2} \mathrm{GlC}-\mathrm{O}-\mathrm{Mg}-\mathrm{O}-\mathrm{Glc}(\mathrm{OH})_{2}$

The reaction of magnesium alkoxide with maltodextrin taken in the 2:1 proportion could result in the products 16-18. The experimental value of $20.1 \%$ of residual magnesium fitted fairly satisfactorily the theoretical value of $20.8 \%$ for compound $\mathbf{1 8}$.

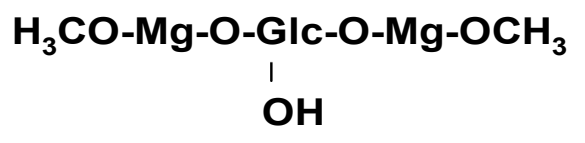

(16)

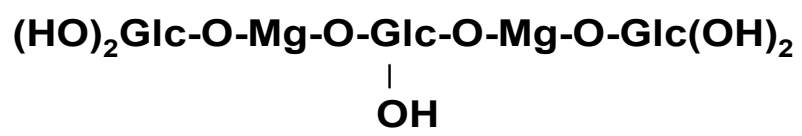

(17)

(HO) ${ }_{2}$ Glc-O-Mg-O-GIC-O-Mg-OCH ${ }_{3}$

।

$\mathrm{OH}$

Maltodextrin as a macroligand did not allow us to decide which among the hydroxyl groups of the D-glucose units were involved in reactions with metal alkoxides. It is likely that, depending on the conformation of the chain in the moment of reaction, all the 2-, 3- and 6-hydroxyl groups of the D-glucose units could participate in the intramolecular reactions. However, in case of compounds such as $\mathbf{2 , 5}$, and $\mathbf{1 3}$, the 3- and 6-hydroxyl groups of the same D-glucose unit have to be involved in the reaction for steric reasons. The lack of selectivity of the hydroxyl groups of maltodextrin in the reactivity and coordination was confirmed by the photoelectron x-ray spectroscopy (XPS) (Table 2). One may see in Table 2 that the ionization energies of the oxygen atoms of the particular hydroxyl groups as well as the oxygen atoms in the glycosidic bonds and within the pyranose rings were very close to one another. In the spectrum of maltodextrin only one fairly broad peak related to the 
ionization energy of all the oxygen atoms could be seen and even the Gaussian analysis of this peak did not separated it into a family of discrete peaks.

The conversion of metal alkoxides into metal maltodextrinates and formation of complexes following that reaction resulted in differentiation of the ionization energies of the oxygen atoms in maltodextrin. Although fundamental spectra invariably showed a single broad peak for the ionization energy common for all oxygen atoms the Gaussian treatment of the peaks provided isolation of discrete peaks.

In the spectra of all postulated compounds, except that of the magnesium complex formed with the 1:1 ratio of magnesium methoxide (13) the additional peaks were discovered on the descending side of the main peaks, this is, on the side of the higher ionization energy of the carbon atom. These peaks confirmed presence of additional carbon atoms in the alkoxide groups introduced into maltodextrin upon their reactions with the metal alkoxides. Changes in the composition of the peak of the ionization energy of the oxygen atoms confirmed more complex differentiation in the status of the oxygen atoms present in the metallated maltodextrins.

Table 2. XPS Spectra of Metal - Maltodextrin Complexes

\begin{tabular}{|c|c|c|c|}
\hline \multirow{2}{*}{$\begin{array}{l}\text { Metal and metal : } \\
\text { D-glucose unit ratio }\end{array}$} & \multicolumn{3}{|c|}{ Ionisation energy $(\mathrm{eV})$} \\
\hline & Metal & Carbon & Oxygen \\
\hline None (maltodextrin) & & $\begin{array}{l}286.70(83.12) \\
287.83(16.88)\end{array}$ & $\begin{array}{l}533.01(99.90) \\
534.73(0.10)\end{array}$ \\
\hline $\mathrm{Bi}(\mathrm{III}), 1: 1$ & $\begin{array}{l}160.58(58.88) \\
165.81(41.12)\end{array}$ & $\begin{array}{l}285.77(12.40) \\
286.70(41.57) \\
288.19(14.45) \\
290.34(12.00) \\
291.72(21.33)\end{array}$ & $\begin{array}{l}530.97(4.85) \\
532.81(73.81) \\
533.96(21.33)\end{array}$ \\
\hline Bi(III), 2:1 & $\begin{array}{l}161.06(56.18) \\
166.37(43.82)\end{array}$ & $\begin{array}{l}286.70(43.57) \\
288.08(11.23) \\
290.02(3.82) \\
292.14(41.39)\end{array}$ & $\begin{array}{l}532.15(7.67) \\
533.49(73.77) \\
534.39(18.57)\end{array}$ \\
\hline
\end{tabular}


Table 2. Cont.

\begin{tabular}{|c|c|c|c|}
\hline $\mathrm{La}, 1: 1$ & $\begin{array}{l}835.57(32.55) \\
839.25(27.33) \\
852.35(21.81) \\
856.06(18.31)\end{array}$ & $\begin{array}{l}286.70(64.95) \\
288.07(17.27) \\
290.89(17.78)\end{array}$ & $\begin{array}{l}530.39(8.59) \\
532.76(91.41)\end{array}$ \\
\hline $\mathrm{La}, 2: 1$ & $\begin{array}{l}835.81(34.38) \\
839.51(25.50) \\
852.59(23.04) \\
856.29(17.08)\end{array}$ & $\begin{array}{l}286.70(66.54) \\
288.07(10.72) \\
290.81(22.73)\end{array}$ & $\begin{array}{l}529.41(6.45) \\
532.57(93.55)\end{array}$ \\
\hline $\mathrm{Mg}, 1: 1$ & $87.10(100.0)$ & $\begin{array}{l}287.30(93.13) \\
287.54(6.87)\end{array}$ & $\begin{array}{l}531.19(4.64) \\
531.82(95.36)\end{array}$ \\
\hline $\mathrm{Mg}, 2: 1$ & $89.03(100.0)$ & $\begin{array}{l}286.70(85.34) \\
287.94(6.59) \\
290.06(8.07)\end{array}$ & $\begin{array}{l}531.82(92.08) \\
533.89(7.92)\end{array}$ \\
\hline
\end{tabular}

${ }^{a}$ Relative percentage of area under given peak is presented in parentheses.

The data in Table 2 for the X-ray spectra of the metal - maltodextrin complexes show that the ionisation energy of the maltodextrin oxygen atoms decreased after the reaction with the bismuth reagent taken at lower proportions. An increase in the proportion of the bismuth reagent used was accompanied by a corresponding increase in the ionization energy.

Regardless of the amount of reagents used on one D-glucose unit, both lanthanation and magnesation caused a decrease in the ionization energy of the maltodextrin oxygen atoms represented by the position of the main band in this region of the spectra. The decrease in the ionization energy did not fall in the order of decreasing Pauling [19] and Sanderson [20] electronegativities of the central atoms. The electronegativities from both sources decreased in the order of $\mathrm{Bi}>\mathrm{Mg}>\mathrm{La}$. This discrepancy can result from an involvement of the hydroxyl groups in the proximity of the metal atoms in the ligation. For steric reasons and because of the conformations of the maltodextrin chain, the coordination sphere of the metal atoms could be only partially filled.

Due to coordination, the metal - oxygen bonds were stabilized against their hydrolysis. Metal derivatives of maltodextrin hydrolyzed slowly even on boiling in water and neither colloids nor gels were formed. Thermogravimetric data (Table 3) showed that bismuthation of maltodextrin reduced its thermal stability with respect to maltodextrin. It decreased with the number of bismuth atoms 
introduced. The initial loss of weight around $100^{\circ} \mathrm{C}$ could result from the evolution of tert-pentanol sorbed or residing in the inner coordination sphere of the Bi central atom in the product. An essential decomposition of bismuthated maltodextrins started at $160^{\circ} \mathrm{C}$, at which point the original maltodextrin also begun to decompose although the amount of products evolved from bismuthated maltodextrins was higher, implying that elimination in the alkoxide moieties also took place at the same stage. That decomposition could influence the thermal stability of the complexes.

In contrast to bismuthated maltodextrins, the corresponding lanthanum and magnesium derivatives were thermally more stable than the original maltodextrin. The derivatisation entirely changed the course of decomposition of the maltodextrin. Both atoms evidently did not contain alcohol ligands in their inner and outer coordination spheres.

Table 3. Thermogravimetric (TG) and Differential Thermogravimetric (DTG) Analysis of Metal Derivatives of Maltodextrins

\begin{tabular}{|c|c|c|c|}
\hline \multirow{2}{*}{$\begin{array}{l}\text { Metal and Metal : D- } \\
\text { glucose Unit Ratio }\end{array}$} & \multicolumn{2}{|c|}{ TG } & \multirow{2}{*}{$\begin{array}{l}\mathrm{DTG}^{\mathrm{a}} \\
\left({ }^{\mathrm{o}} \mathrm{C}\right)\end{array}$} \\
\hline & Effect $\left({ }^{\circ} \mathrm{C}\right)$ & Total Weight Loss (\%) & \\
\hline \multirow{4}{*}{$\begin{array}{l}\text { None, prior to heating } \\
\text { (primary control) }\end{array}$} & 160 & 4.0 & 89 \\
\hline & 240 & 5.5 & \\
\hline & 293.5 & 27.5 & $\mathbf{2 6 4}, 267,271$ \\
\hline & 404 & 39.0 & 409 \\
\hline \multirow[t]{3}{*}{ Bi(III), $1: 1$} & 100 & 11.5 & 80 \\
\hline & 160 & 14.0 & \\
\hline & 300 & 52.0 & 238 \\
\hline \multirow[t]{3}{*}{ Bi(III), 2:1 } & 95 & 8.4 & 70 \\
\hline & 160 & 15.0 & \\
\hline & 270 & 52.0 & $\mathbf{2 0 5}, 255$ \\
\hline \multirow[t]{3}{*}{ La, $1: 1$} & 204.5 & 3.7 & 150 \\
\hline & 260 & 11.8 & $230 \mathrm{~s}$ \\
\hline & 347 & 68.4 & $\mathbf{2 8 5}, 367$ \\
\hline \multirow[t]{3}{*}{ La, 2:1 } & 120 & 4.0 & \\
\hline & 224 & 9.0 & $180 \mathrm{~s}, 240 \mathrm{~s}$ \\
\hline & 350 & 23.7 & $\mathbf{2 9 5}, 360,426$ \\
\hline
\end{tabular}


Table 3. Cont.

\begin{tabular}{|l|l|l|l|}
\hline Mg, 1:1 & 213 & 14.5 & \\
& 264 & 20.7 & 227 \\
& 291.5 & 44.5 & 283 \\
& 310 & 50.7 & \\
& 320.5 & 22.5 & $\mathbf{3 1 6}$ \\
\hline Mg, 2:1 & 205 & 26.6 & 226 \\
& 259 & 41.7 & 287 \\
& 307.5 & 55.4 & $\mathbf{3 1 4}$ \\
\hline
\end{tabular}

a Temperature of the main peak is printed in bold; " $\mathrm{s}$ " denotes a shoulder.

Magnesium salts of maltodextrin were more thermally stable than original maltodextrin. The main decomposition, presented in Table 3 as temperatures of the main DTG peak, were in the case of both magnesium salts approximately $50{ }^{\circ} \mathrm{C}$ higher.

\section{Conclusions}

The results can be summarized as follows:

1. Maltodextrin formed metal derivatives in which metal atoms were bound with involvement of covalent and coordination bonds. Coordination was responsible for the stability of the metal oxygen-carbon moiety towards hydrolysis.

2. Reaction of bismuth tri(tert-pentoxide) with maltodextrin provided compounds $\mathbf{3}$ and $\mathbf{6}$ when the reagents were taken under proportions $1: 1$ and 2:1, respectively. Their thermal stability is lower than that of the original maltodextrin, possibly because of a fairly readily occurring elimination of 2-methylbut-2-ene

3. Reaction of lanthanum triisopropoxide with maltodextrin provided compounds $\mathbf{8}$ and $\mathbf{1 2}$ when the reagents were taken under proportions 1:1 and 2:1, respectively. In both cases, lanthanated maltodextrin was more thermally stable than the original maltodextrin.

4. Reaction of magnesium methoxide with maltodextrin provided compounds $\mathbf{1 3}$ and $\mathbf{1 8}$ when the reagents were taken under proportions 1:1 and 2:1, respectively. These compounds are more thermally stable than the original maltodextrin. 


\section{Experimental}

Bismuthation and Lanthanation of Maltodextrin

Maltodextrin (Maltrin M040; 3.6 kDa, Grain Processing Corp., Muscatine, Iowa, U.S.A) (1.62 g, 0.01 mole of the D-glucose unit) was homogenised in a beaker with either 0.01 or 0.02 mole of metal alkoxide [4.7 and $9.4 \mathrm{~g}$ of bismuth tri-(tert-pentoxide), 3.2 and $6.3 \mathrm{~g}$ of lanthanum tri-isopropoxide, respectively]. Blends were immediately placed in a microwave oven (Whirlpool AKL 535, 800W, Benton Harbor, Missouri, U.S.A) set at medium (50\%) energy and heated for approximately 10 and 6 min. in case of the bismuth and lanthanum reagents, respectively. All reaction products were stored in tightly closed containers.

\section{Magnesium Derivative of Maltodextrin}

Maltodextrin (1.62 g, 0.01 mole of the D-glucose unit) was introduced into 10.8 and $21.6 \mathrm{~g}$ of the magnesium methoxide ( $8 \%$ solution in methanol), respectively. Suspensions of maltodextrin in the magnesium methoxide solution were shaken for 2 days in a closed flask followed by evaporation to dryness under reduced pressure. The reaction products were stored in tightly closed containers.

\section{Behavior of Maltodextrin Metal Derivatives in Contact with Water}

Samples of the reaction products $(0.5 \mathrm{~g})$ were suspended in deionised water $(10 \mathrm{~mL})$ and agitated at room temperature for $30 \mathrm{~min}$. Since samples neither changed their appearance nor any formation of metal hydroxides or oxides were observed, samples were then refluxed for $10 \mathrm{~min}$. No changes in appearance were noted with this treatment either. The $\mathrm{pH}$ of the samples (originally 7.0) did not change after such processing.

Thermal Analysis (Thermogravimetry - TG, Differential Thermogravimetry - DTG, and Differential Thermal Analysis DTA)

Samples $(\sim 100 \mathrm{mg})$ were placed in a corundum crucible and heated in air from room temperature to $500{ }^{\circ} \mathrm{C}$ with a rate of $10{ }^{\circ} \mathrm{C} / \mathrm{min}$. Corrundum, $\phi=8 \mu \mathrm{m}$ was the standard. A computerized PaulikPaulik-Erdey Derivatograph D-1500 Q instrument (Budapest, Hungary) was used.

\section{X-Ray Photoelectron Spectroscopy}

Spectra were recorded with a Physical Electronics Model 5000 Multitechnique Surface Analysis System. The pass energy was $29.350 \mathrm{eV}$ using a Mg source at $300 \mathrm{eV}$. Samples were mounted on 
double-sided adhesive tape. The positions of the peaks were normalised to the position of the main component of the $\mathrm{C} 1 \mathrm{~s}$ band at $286.70 \mathrm{eV}$.

\section{References}

1. Okkerse, C.; van Bekkum, H. Perspectives for a versatile raw material on the threshold of a new millennium, In Starch 96, The Book, Van Doren, H.; Van Swaaij, N., Eds; Carbohydrate Research Foundation: Noordwijkerhout., 1996; Ch.1.

2. Schilling, C.H.; Biner, S.B.; Goel, H.; Jane, J. Plastic shaping of aqueous alumina suspensions with sucrose and maltodextrin additives. J. Environ. Polym. Degr. 1995, 3, 153-160.

3. Kim, J.C.; Auh, K.H.; Schilling, C.H. Effects of polysaccharides on the particle packing and green strength of alumina slurries. Mater. Lett. 2000, 40, 209-212.

4. Kim, J.C.; K.H. Auh, K.H.; Schilling, C.H. Effect of polysaccharides on the rheology of alumina slurries. J. Eur. Ceram. Soc. 2000, 20, 259-266.

5. Kim, J.C; Schilling, C.H.; Tomasik, P.; Auh, K.H. Plasticizing dense alumina slurries with monoand di-saccharides. Mater. Lett. 2000, 40, 221-224.

6. Schilling, C.H.; Li, C.P.; Tomasik, P.; Kim, J.C. The rheology of alumina suspensions: influence of polysaccharides. J. Eur. Ceram. Soc. 2002, 22, 923-931.

7. Chiang, Y.M.; Birnie, D., III; Kingery, W.D. Physical Ceramics, Principles for Ceramic Science and Engineering; John Wiley: New York, 1997; p. 413.

8. Hozer, L. Semiconductor Ceramics, Grain Boundary Effects; Ellis Horwood: New York, 1994; p.11.

9. Lagrange, A. Electronic Ceramics; Steele, B.C.H., Ed.; Elsevier: New York, 1998; p. 1.

10. Lii, C.Y.; Tomasik, P; Yen, M.T.; Hung, W.L.; Lai, V.M.-F. Re-examination of the interactions between starch and salts of metals from the non-transition groups. J. Food Sci. Technol. 2001, 36, 321-330.

11. Ciesielski, W.; Tomasik, P.; Lii, C.Y.; Yen, M.T. Interaction of starch with metal ions from transition groups. Carbohydr. Polym. 2003, 51, 47-56.

12. Baran, W.; Sikora, M.; Tomasik, P.; Anderegg, J.W. Thallium(I) starchates. Carbohydr. Polym. 1997, 32, 209-212.

13. Tyrlik, S.K.; Tomasik, P.; Anderegg, J.W. Titanium(IV) starchate. Carbohydr. Polym. 1997, 34, 17.

14. Tomasik, P.; Schilling, C.H.; Refvik, M.; Anderegg, J.W. Starch - lanthanum complexes. Carbohydr. Polym. 2000, 41, 61-68.

15. Baczkowicz, M.; Wojtowicz, D.; Anderegg, J.W.; Schilling, C.H.; Tomasik, P. Starch complexes with Bismuth (III) and (V). Carbohydr. Polym. 2003, 52, 263-268.

16. Tomasik, P.; Anderegg, J.W.; Jane, J.; Baczkowicz, M. Potato starch derivatives with some chemically bound bioelements. Acta Pol. Pharm., Drug. Res. 2001, 58, 447-452.

17. Marusza, K.; Tomasik, P. Aluminium and arsenic(III) starchates Starch/Staerke 1994, 46, 13-17. 
18. Tomasik, P.; Wang, Y.J.; Spence, K.; Jane, J.; Anderegg, J.W. Starch ferrate. Starch/Staerke 1995, 47, 68-72.

19. Pauling, L. The Nature of the Chemical Bond, $3^{\text {rd }}$ ed.; Cornell University Press: Ithaca, 1960; p. 93.

20. Sanderson, R.T. Inorganic Chemistry; Van Nostrand-Reinhold: New York, 1967; p.72.

Sample Availability: Samples should be freshly prepared.

(C) 2003 by MDPI (http://www.mdpi.org). Reproduction is permitted for noncommercial purposes. 